\title{
Optimising the Pre-Operative Investigative Work Up for Elective Surgical Patients
}

\author{
Nadine Hack-Adams, natalie king, Manisha Ahuja, Simon M Higgs
}

Gloucestershire Hospitals NHS Foundation Trust, United Kingdom

\begin{abstract}
Effective pre-operative assessment of patients awaiting elective surgery should entail appropriate use of scarce NHS resources, as well as underpin patient safety. The pre-operative admissions service in district general hospitals is often junior doctor led, with a new cohort of clinicians taking over its running every four months. Lack of familiarity on the part of these clinicians with the investigative work up required for certain surgical procedures often results in over investigation of patients in the pre-admission setting, wasting time and NHS resources. A retrospective audit of 53 patients who underwent laparoscopic cholecystectomy over a representative two month period demonstrated that $33 \%$ of patients received unnecessary pre-admission blood tests, including clotting screen and 'group and save'. Design and implementation of a "Pre-Admission Handbook", for use by junior doctors and nurse practitioners in the pre-operative setting, reduced the rate of over investigation to $12 \%$ in a subsequent, prospective audit cycle of 50 patients, and has improved patient care by standardising the preadmissions process for elective surgery at Gloucester Royal Hospital.
\end{abstract}

\section{Problem}

The pre-operative admissions service at district general hospitals is often junior doctor led, with a new cohort of clinicians taking over its coordination and running every four months. New to both the surgical department and to clinical practice, these clinicians are often uncertain about the pre-operative investigative work up required for the range of surgical interventions offered by the department, and therefore request a battery of inappropriate and unnecessary tests in the pre admission setting in an attempt to ensure thoroughness. In particular, lack of awareness of the NICE pre-operative guideline for laparoscopic surgery was anecdotally felt to be resulting in a high rate of over investigation of patients awaiting laparoscopic cholecystectomy at Gloucester Royal Hospital. Inappropriate requests for clotting screens and 'group and save' blood tests increase the workload of the hospital laboratories with no clinical gain, as the risk of major bleeding and need for intraoperative transfusion is low for this procedure. This indiscriminate use of resources has clear financial implications in an increasingly resource limited NHS.

\section{Background}

The National Institute for Clinical Excellence (NICE) has published a pre-operative guideline (CG3-2003) which categorises all surgical procedures as minor, intermediate, major or major+, and makes recommendations for appropriate pre-operative investigations according to category of surgery and ASA grade (grade 1: normal, healthy patient; grade 2: patient with mild systemic disease; grade 3: patient with severe systemic disease but the disease is not a threat to life). These guidelines aim to standardise the process of pre-operative investigation across the UK, ensuring that a patient receives a profile of pre-operative investigations which reflects the risk associated with the planned surgical procedure, as well as the comorbidities of the patient. For laparoscopic surgery, such as laparoscopic cholecystectomy, the risk of major blood loss is low, and the guidelines therefore advise against the routine provision of a clotting screen or 'group and save' pre-operatively. This is presumed to result in effective stewardship of scarce NHS resources without compromising patient safety.

\section{Baseline measurement}

Departmental compliance with NICE guidelines for the preoperative assessment of patients awaiting intermediate surgery (Guideline CG3 - 2003) was audited, using laparoscopic cholecystectomy as a representative example of intermediate surgery.

A list of patients who underwent laparoscopic cholecystectomy from December 2013 to January 2014 was obtained from departmental secretaries (53 patients in total). The Gloucestershire 'PAS' Interface was used to determine which investigations were ordered at pre-admission clinic; in particular, whether clotting screen and 'group and save' were requested. Medical notes were pulled from Medical Records at Gloucester Royal and reviewed to determine whether the clinic was junior doctor or nurse practitioner led, whether an intra-operative transfusion was required, and whether the procedure was cancelled due to lack of clotting screen or group and save. No patient in the cohort was taking oral anti-coagulants at the time of audit.

Over the two month period, the percentage of patients undergoing laparoscopic cholecystectomy who received an unnecessary clotting screen or 'group and save', against the recommendations of the guideline, was determined. The question of whether patient safety was compromised or care delayed by lack of availability of these blood tests at the time of surgery was also addressed; in particular the percentage of patients requiring an intra-operative 
transfusion or for whom the procedure was cancelled due to lack of these investigations was recorded.

As shown in the supplementary material, $25 \%$ of patients $(n=13)$ received a clotting screen, whilst $33 \%$ of patients $(n=17)$ received a 'group and save', against the recommendations of the guideline. No patient required an intra-operative transfusion and no procedures were cancelled due to the lack of availability of these blood tests. In $80 \%$ of cases, these tests were requested by the junior doctors running the clinic.

See supplementary file: ds3984.xlsx - "Pre Handbook"

\section{Design}

When considering the underlying reasons for over investigation in pre-admission clinic, it became clear that junior doctors were not aware of the existence of the NICE guidelines and, in any case, would not have time to read and assimilate this lengthy document in the busy clinical environment in which they work. Junior doctors were also not familiar with the expectations of departmental consultants with regard to pre-operative assessment, leading to concerns about "missing something" or delaying a patient's operation if all the necessary blood tests were not available on the day of surgery. The departmental consultants were therefore surveyed in the first instance and asked to provide a list of the preoperative investigations which they felt to be essential for each surgical procedure they performed. This list was then compared with the recommendations from NICE CG3-2003. A Pre-Admissions Handbook was developed, which aimed to summarise departmental preference and the NICE guidelines in the form of a succinct, readable document which junior doctors could take into the clinic room with them as an aide memoir when pre-admitting elective surgical patients.

\section{Strategy}

PDSA Cycle 1: The pre-admission handbook was initially shown to the consultant surgeons, as well as the advanced nurse practitioners within the department. All departmental consultants and nurse practitioners were invited to rate the handbook across several categories: overall appearance, organisation of information, readability, and accuracy of information. The reviewers were satisfied with the overall appearance and layout of the document, but recommended the addition of further information for each surgical procedure, including a brief description of each surgical procedure, prior to a trial with the end users.

PDSA Cycle 2: The pre-admission handbook was trialed on the departmental surgical house officers after a summary for each surgical procedure had been added. The feedback from the testing was positive; the surgical house officers felt much more confident in requesting the pre-operative investigations for all procedures performed by the department, and commented that it was reassuring to know that they were acting in compliance with local preference and national guidelines. However, they also commented that the lack of a contents page made it difficult to flick through the document to the correct section in the middle of a busy clinic.

PDSA Cycle 3: In this cycle, the handbook was re-organised by category of surgical intervention (minor/intermediate/major/major+) and a contents section was added. The handbook was then trialled again with the same population of surgical house officers. This time there was no negative feedback; the doctors commented that the handbook was now much easier to use, with appropriate and intuitive ordering of information and a clearly laid out contents page.

PDSA Cycle 4: The handbook was then trialled for two months in the surgical pre-admissions clinic (April-May 2014) and the same end points were audited as before. Feedback continued to be positive - no negative feedback at this stage.

PDSA Cycle 5: The pre-admission handbook was rolled out across the surgical department at Gloucester Royal after presentation of the results of the audit from PDSA 4 at the trustwide Clinical Governance meeting in August 2014.

PDSA Cycle 6: The pre admission handbook is currently in use by the August cohort of foundation year one doctors, with a re-audit of the same endpoints planned for October/November 2014.

\section{Results}

Post intervention measurement covered a representative two month period after the handbook had been introduced and looked at the same end points as before (i.e. percentage of patients awaiting laparoscopic cholecystectomy who received inappropriate clotting screens and 'group and saves'; whether any operations were cancelled because of the lack of availability of the necessary blood tests, and whether any laparoscopic cholecystectomy patients required an intra-operative transfusion). 50 patients were audited over this period. The findings were that 6 patients $(12 \%)$ received an unnecessary clotting screen and the same $6(12 \%)$ an unnecessary group and save in pre admission clinic, compared with $25 \%$ and $33 \%$ respectively prior to the introduction of the handbook, demonstrating a $50-60 \%$ reduction in the rate of over investigation in the pre operative setting after the introduction of the handbook. It was also worthy of note that none of these tests were requested by junior doctors in this audit cycle.

However, closer appraisal of each patient record on the PAS interface revealed that 20 patients from this cohort (40\%) went on to receive an unnecessary 'group and save' on the day of their laparoscopic cholecystectomy, when they attended the nurse led surgical admissions suite. This will be discussed further in the 'Lessons and Limitations' section which follows.

See supplementary file: ds3982.xlsx - "Post Handbook"

\section{Lessons and limitations}

A number of lessons were learnt during the planning and implementation of this quality improvement project. Firstly, use of the PDSA cycle to plan and implement small changes at each stage 


\section{BMJ Quality Improvement Reports}

in the project was a really useful way of making a change and then assessing its impact in a structured and logical way. Dialogue with 'stake holders' (i.e. consultants, advanced nurse practitioners, and junior doctors) was an essential part of the process at every stage, without which the pre admissions handbook could not have been developed or satisfactorily trialled.

The realisation that a high proportion of patients were still receiving unnecessary blood tests in the surgical admissions suite on the day of their procedure, or at the request of the anesthetist running the list, was frustrating. It demonstrates that a change in culture for the better in one department does not necessarily translate automatically to a change in practice in other, closely related departments. For lasting change to affect an entire trust, there needs to be inter and intra-departmental engagement with the process of service improvement and communication at all levels, from junior doctors and nurses, to consultants and chief executives. Without this, a quality improvement project and its successes will always be limited in their extent and impact.

Subsequent PDSA cycles would ideally include dialogue with the senior sisters in the surgical admissions suite, with attempts made to engage stakeholders in this department with the quality improvement process and reduce the amount of over investigation happening on the day of surgery.

\section{Conclusion}

Post design and distribution of the Pre Admissions Handbook, departmental awareness of best practice guidelines for preoperative assessment of elective surgical patients has improved. Fewer patients are being inappropriately over investigated prior to undergoing elective laparoscopic surgery, which is of financial significance in an increasingly resource limited National Health Service. Importantly, patient safety is not compromised by the rationalisation of the investigative work up; no intra-operative transfusions were required during an elective laparoscopic cholecystectomy in the time periods audited, and no elective laparoscopic cholecystectomies were cancelled due to lack of clotting/group and save. There are plans in place to complete further PDSA cycles to encourage lasting change within the department and promote awareness of best practice guidelines in other departments, notably the nurse led surgical admissions suite, to ensure that improvements in service provision happen across the trust rather than just within one department in isolation. The educational value of the project is also worthy of mention; new junior doctors feel more empowered to run pre-admission clinic in the knowledge that they are complying with national guidelines and are contributing to effective stewardship of NHS resources, without compromising patient safety.

\section{References}

1. Reeves B, Emberton M, Thoms G. Pre Operative Tests: the use of routine preoperative tests for elective surgery. NICE Guidelines (CG3). June 2003.

\section{Declaration of interests}

None declared.

\section{Acknowledgements}

Gaynor Jones (Advanced Nurse Practitioner)

\section{Ethical approval}

This project was a quality improvement study, which aimed to ensure consistent implementation of accepted best practice, based on NICE guidelines. 